\title{
Dimensions of pes anserinus of the lower extremity, an anatomical study with its surgical implications
}

\author{
Rajanigandha Vadgaonkar, ${ }^{1,2}$, M.D. Prameela ${ }^{1,2}$, Chettiar Ganesh Kumar ${ }^{1,2}$, Vandana Blossom ${ }^{1,2}$, \\ Mamatha Tonse ${ }^{1,2}$, B.V. Murlimanju ${ }^{1,2}$, Mangala M. Pai ${ }^{1,2}$, Latha V. Prabhu ${ }^{1,2}$ \\ ${ }^{1}$ Department of Anatomy, Kasturba Medical College, Mangalore, ${ }^{2}$ Manipal Academy of Higher Education, Manipal, Karnataka, India
}

\begin{abstract}
The reconstructive surgeries utilize pes anserinus (PA) tendons, because of their lesser post-operative clinical deficits and donor site morbidity. These surgeries require anatomical knowledge about the extent of PA formation. The goal of this study was to determine the length and width of the PA formation. The objectives were to measure the distance of its upper limit, lower limit, and vertical distance from the tibial tuberosity (TT). The present descriptive cross sectional study included 53 embalmed cadaveric lower extremities. The upper and lower limits of PA were exposed with the careful dissection. Measurements of the dimensions were performed with the help of a digital vernier caliper (Mitutoyo Co., Kanagawa, Japan). The PA length, width, distance of its upper limit, lower limit, and vertical distance of it from the TT were $47.4 \pm 13.3 \mathrm{~mm}, 37.3 \pm 7.2 \mathrm{~mm}, 47.6 \pm 12.5 \mathrm{~mm}, 54.6 \pm 10.4 \mathrm{~mm}$, and $39.1 \pm 14.2 \mathrm{~mm}$, respectively over the right extremity. The same measurements were $46.3 \pm 14.7 \mathrm{~mm}, 39.1 \pm 9.4 \mathrm{~mm}, 39.1 \pm 5.9 \mathrm{~mm}, 49.5 \pm 8.2 \mathrm{~mm}$, and $36.4 \pm 12.1 \mathrm{~mm}$, respectively for the left extremity. The extent of PA was observed to be extremely variable. The preoperative knowledge about the dimensions of PA will help the plastic and orthopedic surgeon put the accurate skin incision, decreasing the donor site morbidity and biomechanical instability of the PA grafts. We suggest that, preoperative ultrasound measurement of the PA may help the operating surgeon to prevent the complications like injury to the infrapatellar branch of saphenous nerve.
\end{abstract}

Key words: Transplants, Reconstructive surgical procedures, Tibia

Received October 22, 2020; Revised January 7, 2021; Accepted February 23, 2021

\section{Introduction}

The guy ropes in the lower extremity form a tent-like structure, which provides strength to the pelvis. These also give the femur and tibia stability in weight bearing, balance and mobility of lower limb. Sartorius (SA), Gracilis (GC) and semitendinosus (ST), which belonged to the muscles of

\section{Corresponding author:}

B.V. Murlimanju (iD

Department of Anatomy, Kasturba Medical College, Manipal University, Mangalore 575004, India

E-mail: flutesnowmm@gmail.com anterior, medial, and posterior compartments of the thigh, form the guy ropes. The guy ropes insert together, and this radiating arrangement resembles the 'goosefoot'. Hence it is given the name 'pes anserinus (PA)' $[1,2]$. The tendons vary in their dimensions and insertion, which leads to altered dimensions of the PA. PA is nothing but the confluence of musculotendinous structures [3]. The surgical excision of PA is a common practice in constructive procedures like the repair of tendocalcaneus, repair of the ruptured patellar tendon and subluxation, anterior cruciate ligament reconstruction, and PA transplantation during surgical grafting [1-2, 4]. The painful knee is a common complaint in the orthopedic practice, and PA syndrome had been frequently diagnosed [5]. The PA is preferred for the reconstructive surgeries due to 
their less morbidity at the donor site and there are no postoperative deficits [6]. It was reported that anatomical variations are among the causes for the complications, associated with PA tendon grafting [7]. The minimally invasive surgical procedures require an understanding of the exact location of the insertion of PA [8]. The clinical implications are high, but the studies are scarce in the literature about PA's dimensions. The present anatomical research goal was to determine the length and width of PA formation over the right and left lower extremities. The objectives were to measure the distance of its upper limit, lower limit and vertical distance from the tibial tuberosity (TT) in the cadaveric lower limb specimens.

\section{Materials and Methods}

This anatomical investigation comprised 53 embalmed adult human cadaveric lower extremities, of which 27 were right-sided, and 26 left-sided. The present study did not take the gender of specimens into consideration. Present study excluded the samples which had pathological changes, congenital anomalies, and destructions. This study has the approval of our institutional ethics committee. All the samples followed meticulous dissection and the PA was exposed by reflecting the skin and fascia at the supero-medial aspect of the leg's anterior compartment. The dimensions were measured using the digital Vernier caliper of accuracy, \pm 0.01

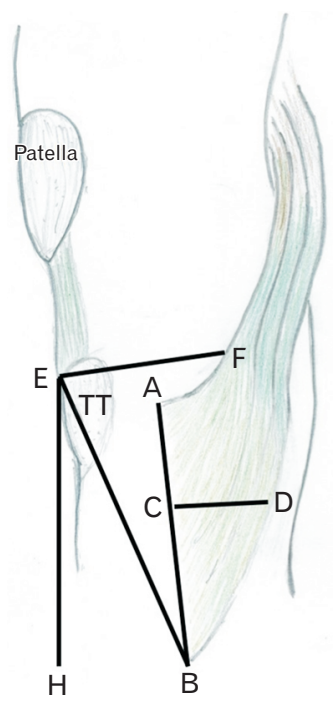

Fig. 1. Schematic representation of the measurements performed in the present study. $A B$, pes anserinus length; $C D$, pes anserinus width; $\mathrm{EB}$, distance of its lower limit from TT; EF, distance of its upper limit from the midpoint of TT; EH, vertical distance of its lower limit from the midpoint of TT; TT, tibial tuberosity. inch (model-500 196; Mitutoyo Co., Kanagawa, Japan), and tabulated separately for the right and left sides. The data were analyzed using IBM SPSS Statistics for Windows, Version 25.0 (IBM Co., Armonk, NY, USA), measurements are given in millimeters and represented as mean \pm standard deviation (SD). The paired $t$ test was applied to compare the right and left side measurements. If the $P$-value is smaller than 0.05 , the comparison was considered statistically significant.

The present study measured the following dimensions of PA:

1. The PA length (AB in Fig. 1)

2. The width of PA (CD in Fig. 1)

3. The distance of its upper limit from the TT (EF in Fig. 1)

4. The distance of its lower limit from the TT (EB in Fig. 1)

5. The vertical distance of its lower limit from the TT (EH in Fig. 1)

The PA length $(\mathrm{AB})$ was the distance between the superior and inferior limit (Fig. 2) of the PA's distal attachment into the supero-medial aspect of the tibia. The anteroposterior extent (Fig. 2) of the PA formation at its mid-level was considered as the PA's width (CD). The distance of the upper limit of PA was measured from the mid-point of TT (EF). The upper limit of PA was the proximal point at which all the three constituent tendons of PA were converging (Fig. 2). Similarly, the lower limit of PA was also measured from

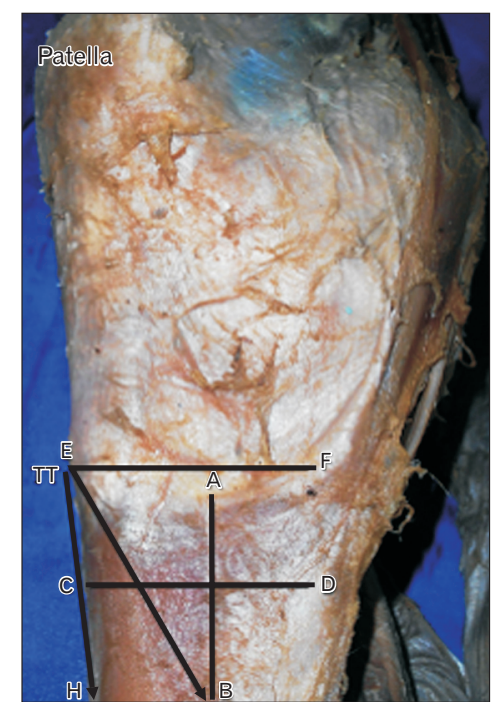

Fig. 2. The photograph of the cadaveric lower limb showing the characteristics of pes anserinus and the measurements performed in the present study. $A B$, pes anserinus length; $C D$, pes anserinus width; $\mathrm{EB}$, distance of its lower limit from TT; EF, distance of its upper limit from the midpoint of TT; EH, vertical distance of its lower limit from the midpoint of TT; TT, tibial tuberosity. 
Table 1. Dimensions of PA observed in the present study $(n=53)$

\begin{tabular}{lc}
\hline \multicolumn{1}{c}{ Dimension } & Measurement (mm) \\
\hline PA length & $46.7 \pm 14.4$ \\
PA width & $38 \pm 8.5$ \\
Distance of upper limit of PA from TT & $43.8 \pm 10.8$ \\
Distance of lower limit of PA from TT & $52.3 \pm 9.9$ \\
Vertical distance of lower limit of PA from TT & $37.9 \pm 13.5$ \\
\hline
\end{tabular}

Values are presented as mean \pm SD. PA, pes anserinus; TT, tibial tuberosity; SD, standard deviation.

the mid-point of TT (EB). This was the distal point in the tibia, where PA formation was attaching (Fig. 2). The vertical distance of the lower limit of PA from TT (EH) was the measurement performed between the mid-point of TT and the point on the anterior border (shin) of the tibia, which was located at the same level as the lower limit of PA (Fig. 2).

\section{Results}

The dimension of PA was observed to be extremely variable. The PA was often merging with the facia cruris (FC) and extending very much inferior, which gave a greater extent of the PA supero-inferiorly. Table 1 represents the dimensions of PA and also provides the mean data of all the specimens, which are included in this study. The dimensions of the PA for the right and left lower extremity are represented separately in Table 2 . The statistical comparison of PA length, width, and vertical distance from the TT did not reveal a significant difference over the left and right sides $(P>0.05)$. However, the dimensions were higher for the rightsided PA in the distance of its upper and lower limits from the TT $(P<0.05)$, in comparison to the left side (Table 2$)$.

\section{Discussion}

The PA is composed of tendons of SA, GC, and ST muscles. The three muscles of PA form the elements from each compartment of the thigh. SA begins from the anterior superior iliac spine and represents the muscle of the front of the thigh. GC begins from the ischiopubic ramus and symbolizes the adductor compartment. The third muscle, ST, originates from the upper part of the ischial tuberosity and embodies the muscle of the back of the thigh [9]. However, our previous study [10] observed that semimembranosus muscle was taking part in the PA formation in $5.7 \%$ of cases. The ST was giving an extra slip, which was attaching to the medial condyle of the tibia in $13.2 \%$ cases, to the medial col-
Table 2. Sidewise comparison of the dimensions of PA $(n=53)$

\begin{tabular}{|c|c|c|c|}
\hline Dimension & $\begin{array}{l}\text { Right side } \\
\quad(\mathrm{n}=27)\end{array}$ & $\begin{array}{l}\text { Left side } \\
(n=26)\end{array}$ & $P$-value \\
\hline PA length & $47.4 \pm 13.3$ & $46.3 \pm 14.7$ & 0.7 \\
\hline PA width & $37.3 \pm 7.2$ & $39.1 \pm 9.4$ & 0.4 \\
\hline Distance of upper limit of PA from $\mathrm{TT}^{\star}$ & $47.6 \pm 12.5$ & $39.1 \pm 5.9$ & 0.004 \\
\hline Distance of lower limit of PA from TT ${ }^{*}$ & $54.6 \pm 10.4$ & $49.5 \pm 8.2$ & 0.03 \\
\hline Vertical distance of lower limit of PA from TT & $39.1 \pm 14.2$ & $36.4 \pm 12.1$ & 0.2 \\
\hline \multicolumn{4}{|c|}{$\begin{array}{l}\text { Values are presented as mean } \pm \mathrm{SD} \text {. PA, pes anserinus TT, tibial tuberosity SD, } \\
\text { standard deviation. The measurements are given in millimetres. The paired } t \\
\text { test was applied for the statistical analysis. }{ }^{*} \text { The difference was considered as } \\
\text { statistically significant if the } P \text {-value is less than } 0.05 \text {. }\end{array}$} \\
\hline
\end{tabular}

lateral ligament in 3.8\% cases, and ligamentum patellae in $1.9 \%$ cases [10]. Femoral, obturator and tibial nerves supply the SA, GC and ST respectively and these muscles get the nourishment by the muscular branches of femoral, profunda femoral, medial circumflex femoral, inferior gluteal, and perforating arteries [9]. SA performs the flexion of knee and hip joints, along with the abduction and external rotation of hip joint. GC causes flexion, medial rotation, and adduction of the hip. ST helps the knee joint's flexion, medial rotation of the leg and weak extension of the hip.

The PA is inferomedially related to the knee joint, and the infrapatellar branch of the saphenous nerve. The morphological information of the structures, located medial to the knee joint, the relation between the FC and PA, is important for the clinical diagnosis and management of orthopaedic ailments [2]. In this anatomical study, PA's dimensions were determined to obtain the knowledge about the extent of this tendinous intersection. If the PA merges with FC, its length will be greater. The tendons of ST and GC have aponeurotic ends, which are observed to be joining with the FC [2]. Sometimes FC may be attached to the tibial collateral ligament and fascia covering the medial head of the gastrocnemius.

The data of the present study was compared with the previous reports, which are available in the literature. Ashaolu et al. [4] performed measurements for the PA's distances from the inferior border of TT. In their study, the minimum horizontal distance was $24.96 \mathrm{~mm}$, and the maximum was $65.03 \mathrm{~mm}$. The lowest insertion point was located 124.44 $\mathrm{mm}$ from the TT. In our research, these dimensions were $43.8 \pm 10.8 \mathrm{~mm}, 52.3 \pm 9.9 \mathrm{~mm}$, and $37.9 \pm 13.5 \mathrm{~mm}$, respectively. Ashaolu et al. [4] reported that the PA's breadth and length in their study was $134.02 \mathrm{~mm}$ and $40.36 \mathrm{~mm}$ respectively. In a Thai population study, the distance between TT and PA 
insertion was $5 \mathrm{~mm}$ at the vertical plane and $25 \mathrm{~mm}$ at the horizontal plane [11]. In the present study, these dimensions were $38 \pm 8.5 \mathrm{~mm}$ and $46.7 \pm 14.4 \mathrm{~mm}$, respectively. The differences in the dimensions of the data between our study and previous reports $[4,11]$ are due to the differences in the topographical points considered to perform the measurements. The differences may also be due to ancestral variations as the present study included samples from Indian population and included samples from Nigerian [4] and Thai [11] population.

Only few studies are available in the literature about the PA dimensions and are not documented in the Indian literature. The scientific literature can consider this anatomical study as novel from Indian samples. This study has measured the length and breadth of the PA in cadaveric specimens. Since, TT is an important landmark, its distance from the PA was recorded. The distance was measured between the TT and the upper and lower limits of PA and also the vertical distance of PA from the TT. The lack of PA's anatomical knowledge can lead to difficulty in topographical identification of its insertion during the surgery [12] and ineffective small sized grafts [13]. It was reported that, $46.8 \%$ of patients who are suffering from osteoarthritis of the knee, had inflammation of the anserine bursa [8]. The diagnosis of bursitis syndrome and PA tendinitis should be considered in cases of pain at the PA insertion site. The local steroid injection can treat the bursitis, but this requires topographical knowledge about the bursa and PA insertion [8]. The anterior cruciate ligament grafts from the ST and GC tendons can be compared to the patellar tendon-bone graft because of the equal cross-sectional area [14]. The adequately executed PA transplant is crucial in preventing the post-operative anterior-external tibial rotation and valgus deformity [12].

Munhoz et al. [13] reported that the distance between PA and TT varies from person to person. The dimensions like length, width, thickness and tibial attachment of PA can be estimated using ultrasound technology. The ultrasound plays an essential role in diagnosing PA diseases. The infrapatellar branch of the saphenous nerve is prone to get injured while harvesting the PA. The average distance between the infrapatellar nerve and the PA is $95 \mathrm{~mm}$ [15]. Zhong et al. [15] opined that the preoperative ultrasound could help the operating surgeons avoid the damage to the saphenous nerve. The preoperative measurement of PA dimensions by using the radiological methods is useful to the operating surgeon [10]. The ultrasound is the best method to observe the PA morphology because of its proximity to the skin [15]. The morphometric data about PA are scarce from the global literature. In the routine dissection teaching, the typical presentation of the musculature is always emphasized [16]. But there is a need for the knowledge about the variant anatomy of these muscles. We have reported the morphological variants of the PA formation in our previous study [10].

Ugai et al. [17] reported that, morphological alteration of the PA tendon could lead to the formation of bony spurs. Ivey and Prud'homme [18] dissected the tendons of PA, by an inverted ' $\mathrm{L}$ ' subperiosteal incision, beginning at the crest of the tibia. They observed that, tendons of GC and ST fuse together at $31.8 \mathrm{~mm}$ away from their insertion site and later fused with the SA tendon. Ivey and Prud'homme [18] also observed fascial loops connecting the SA tendon with the tibial collateral ligament in about $48 \%$ of their cases. The PA had each of the three tendons inserting separately into either tibia or fascia cruris. Ivey and Prud'homme [18] suggested that, inverted 'L' subperiosteal incision, which begins at the crest of the tibia can dissect the tendons of the PA for the clinical use in reconstructive procedures of the knee, effectively and safely. ST and GC tendons are often used for the ACL reconstruction. But it is not uncommon to find the accessory tendinous slips arising from these two tendons, while harvesting the grafts [6]. These tendinous slips usually originate $10 \mathrm{~cm}$ beyond the proximal part of the tendon. Candal-Couto and Deehan [6] suggested that, extra care should be taken to avoid cutting the main tendon. This can be accomplished by using a tendon stripper and it is essential to look for these accessory slips as the anatomy of these bands is more variable than reported earlier [6].

PA forms complex multi-layered stabilising structure, which supports the medial component of the knee in the erect position $[2,19]$. The insertion of PA is into the posteromedial aspect of tibia, $42 \pm 7 \mathrm{~mm}$ below the level of the tibial condyle. The insertion site is infero-medial to the TT. But the topographical location of the insertion site of PA is extremely variable, due to the presence of accessory slips [20]. Injuries to the PA can involve whole of it or one of its constituting tendons [19]. The additional slips are also known as vincula, which have variable insertion sites like gastrocnemius aponeurosis and surrounding connective tissue layers $[7,20]$. The vincula from the posterior aspect of PA into the popliteal fossa, may insert into the popliteal fascia and the medial head of the gastrocnemius muscle [20,21]. If these insertions remain intact and the main tendon has been cut, 
there is a chance of pulling of the muscles into the posterior aspect of the knee. The detached main tendon may become fibroses, and this may cause disability and pain around the knee joint $[20,21]$. The routine MRI of knee will not include the insertion of the distal part of the PA. This may make the diagnosis of the injuries, difficult as the diagnosis based only on the history and the clinical examination is hard. It is suggested that the scanning should be extended to include the full extent of all the PA tendons up to their insertion [20]. This is particularly important in cases of traumatic injuries of the knee region, whenever there is a high fluid signal in the popliteal fossa. If the pathological condition is not clearly recognised and managed, it will result in poor outcome of the patient with reduced joint function, disability and pain [21].

In this present study, the dimensions of PA were estimated by performing the measurements. This study's future implication is that the anatomical data can be compared with the radiological methods of the PA morphometry. This anatomical study's limitation included the gender based comparison of the PA dimensions, which was not performed. The larger sample size can interpret the morphometric data of this study, still better. The cadaveric specimens used in this study were embalmed and may not give accurate measurements. However, this study's strength is that this is the first reported Indian anatomical study. The morphological database for the Indian population could be obtained from the data of this study.

In conclusion, the dimension of the PA was observed to be extremely variable. We believe that this study's morphometric data will help the operating surgeon put the accurate skin incision, decrease the donor site morbidity, and decrease the biomechanical instability of the PA grafts. The preoperative knowledge about PA's dimensions may help the plastic and orthopaedic surgeons prevent intraoperative complications like injury to the infrapatellar branch of saphenous nerve. The preoperative assessment of PA by the ultrasound measurement is suggested.

\section{ORCID}

\author{
Rajanigandha Vadgaonkar: \\ https://orcid.org/0000-0001-5577-495X \\ M.D. Prameela: https://orcid.org/0000-0001-6581-6631 \\ Chettiar Ganesh Kumar: \\ https://orcid.org/0000-0002-8737-1909
}

Vandana Blossom: https://orcid.org/0000-0003-0129-9916

Mamatha Tonse: https://orcid.org/0000-0003-1322-6730

B.V. Murlimanju: https://orcid.org/0000-0003-1248-8296

Mangala M. Pai: https://orcid.org/0000-0001-6995-5807

Latha V. Prabhu: https://orcid.org/0000-0002-1386-0099

\section{Author Contributions}

Conceptualization: BVM. Data acquisition: RV, MDP, CGK, VB, MT. Data analysis or interpretation: BVM. Drafting of the manuscript: BVM. Critical revision of the manuscript: MMP, LVP. Approval of the final version of the manuscript: all authors.

\section{Conflicts of Interest}

No potential conflict of interest relevant to this article was reported.

\section{Acknowledgements}

The authors sincerely thank the body donors of the corpses; this research had utilized. We appreciate their help towards medical teaching and research.

This paper was presented at the '47th ASSA Conference 2019-Anatomical Society of Southern Africa Conference' which was held in Pilanesberg, South Africa during 7th to 10th April 2019.

\section{References}

1. Amatuzzi MM, Cocco LF, Di Dio LJ, Gotfryd AO. Surgical anatomy of the variations of the arrangement of the tendons of the muscles of the pes anserinus in male adults. Ital J Anat Embryol 2002;107:29-35.

2. Mochizuki T, Akita K, Muneta T, Sato T. Pes anserinus: layered supportive structure on the medial side of the knee. Clin Anat 2004;17:50-4.

3. Vadgaonkar R, Prameela MD, Murlimanju BV, Tonse M, Kumar CG, Massand A, Blossom V, Prabhu LV. Morphometric study of the semitendinosus muscle and its neurovascular pedicles in South Indian cadavers. Anat Cell Biol 2018;51:1-6.

4. Ashaolu JO, Osinuga TS, Ukwenya VO, Makinde EO, Adekanmbi AJ. Pes anserinus structural framework and constituting tendons are grossly aberrant in Nigerian population. Anat Res Int 2015;2015:483186.

5. Helfenstein M Jr, Kuromoto J. Anserine syndrome. Rev Bras Reumatol 2010;50:313-27. 
6. Candal-Couto JJ, Deehan DJ. The accessory bands of Gracilis and Semitendinosus: an anatomical study. Knee 2003;10:325-8.

7. Olewnik Ł, Gonera B, Podgórski M, Polguj M, Jezierski H, Topol M. A proposal for a new classification of pes anserinus morphology. Knee Surg Sports Traumatol Arthrosc 2019;27:2984-93.

8. Lee JH, Kim KJ, Jeong YG, Lee NS, Han SY, Lee CG, Kim KY, Han SH. Pes anserinus and anserine bursa: anatomical study. Anat Cell Biol 2014;47:127-31.

9. Standring S. Gray's anatomy: the anatomical basis of clinical practice. 41st ed. New York: Elsevier Limited; 2016. p. 1361-6.

10. Murlimanju BV, Vadgaonkar R, Ganesh Kumar C, Prameela MD, Tonse M, Pai MM, Blossom V. Morphological variants of pes anserinus in South India. Muscles Ligaments Tendons J 2019;9:372-8.

11. Kijkunasathian C, Limitlaohaphan C, Saengpetch N, Saitongdee $\mathrm{P}$, Woratanarat $\mathrm{P}$. The location of pes anserinus insertion in Thai people. J Med Assoc Thai 2009;92 Suppl 6:S189-92.

12. Slocum DB, Larson RL, James SL. Pes anserinus transplant: impressions after a decade of experience. J Sports Med 1974;2:12336.

13. Munhoz MAS, Cunha FB, Mestriner G, Ferreira FC, Leme RV, Galdeano EA, Alcaraz AM, Cunha MR. Anatomical and morphometric study of the pes anserine tendons in the knee. J Clin Diagn Res 2018;12:RC05-7.
14. Tohyama H, Beynnon BD, Johnson RJ, Nichols CE, Renström PA. Morphometry of the semitendinosus and gracilis tendons with application to anterior cruciate ligament reconstruction. Knee Surg Sports Traumatol Arthrosc 1993;1:143-7.

15. Zhong S, Wu B, Wang M, Wang X, Yan Q, Fan X, Hu Y, Han Y, $\mathrm{Li} Y$. The anatomical and imaging study of pes anserinus and its clinical application. Medicine (Baltimore) 2018;97:e0352.

16. DeLuca MK, Boucher LC. Morphological variations and accessory ossicles in the peroneal and tibialis muscles. Anat Cell Biol 2019;52:344-8.

17. Ugai K, Sato S, Matsumoto K, Matsubara T, Mizuno K, Hirohata K. A clinicopathologic study of bony spurs on the pes anserinus. Clin Orthop Relat Res 1988;(231):130-4.

18. Ivey M, Prud'homme J. Anatomic variations of the pes anserinus: a cadaver study. Orthopedics 1993;16:601-6.

19. Albtoush OM, Taib AA, Horger M, Springer F. Avulsion fracture of an ossified pes anserinus tendon post-lateral patellar dislocation. Skeletal Radiol 2018;47:699-702.

20. Ariyaratne SP, Clarnette R, Loftus WK. A rare case of isolated traumatic avulsion of the pes anserinus tendon. Skeletal Radiol 2020;49:1659-62.

21. Cooper DE, Conway JE. Distal semitendinosus ruptures in elite-level athletes: low success rates of nonoperative treatment. Am J Sports Med 2010;38:1174-8. 